Proceedings

\title{
The impacts of green space structure on asthma in Toronto, Canada
}

\author{
Yuping Dong ${ }^{1,2}$, Helin Liu ${ }^{1,2, *}$ and Tianming Zheng ${ }^{1,2}$
}

\author{
${ }^{1}$ School of Architecture and Urban Planning, Huazhong University of Science and Technology, Wuhan 430074, \\ China; youkie@hust.edu.cn (Y.D.); tm1996@hust.edu.cn \\ ${ }^{2}$ Center for Urban and Rural Planning Support Research, Huazhong University of Science and Technology, \\ Wuhan 430074, China \\ * Correspondence: Correspondence: hl362@hust.edu.cn; Tel.: +86-1552-771-9130
}

Publisher's Note: MDPI stays neutral with regard to jurisdictional claims in published maps and institutional affiliations.

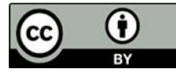

Copyright: (c) 2020 by the authors. Submitted for possible open access publication under the terms and conditions of the Creative Commons Attribution (CC BY) license (http://creativecommons.org/licenses/by/4.0/).

\begin{abstract}
A series of studies have proved that green space can influence air pollution, which is the main risk factor for asthma. In addition, the biodiversity hypothesis suggests use of green space can contribute to increasing human commensal microbiota on the skin, which is possible to enhance immunity to allergic atopy. Given that, the co-effects of green space might jointly influence asthma. However, existing studies mainly focus on one or part of the pathway between them, and most of them shed light on the influence of greenness or trees on asthma. Green space structure, capable to influence both air pollution and microbial diversity, is also probably a significant factor to influence asthma. Regarding this, this study takes Toronto as the case to explore two potential mechanisms that shape the impact of green space structure on asthma. Besides, tree diversity that might moderate this impact was also examined in this study. By regression analysis, it is found that the impact of green space structure on the prevalence of asthma by reducing air pollution was not evident. Moreover, green space structure has no significant contribution to the prevalence of asthma, but when moderated by tree diversity, it has the potential to reduce the prevalence of male asthmatics and such moderation effect only works on male asthmatics. Except for that, several covariates selected in this study were also found correlate with the prevalence of asthma. In particular, the relationship between household income and the prevalence of asthma is only significant among female asthmatics.
\end{abstract}

Keywords: Green space structure; Impacts; Asthma; Air pollution; Tree diversity; Toronto

\section{Introduction}

Asthma is an airway inflamed disease and could induce breathing difficulty and airflow obstructed by inflaming and narrowing bronchial tubes. It has become the third-most chronic disease in Canada [1]. As reported, there are about 300 people diagnosed as asthmatics every day in Canada, and it causes approximately 250 death every year [1]. Furthermore, an online investigation suggests that asthma has serious influences on patients' physical, mental, and social health [2]. For example, most respondents point out that, except for the physical damages, asthma additionally makes them feel anxious, hopeless, stigmatized, and less confident. Although the prevalence of asthma might be impacted by various elements, like urbanization, allergens, gender, age, genes, etc., inhaled particles as well as substances are considered the strongest risk factors [3]. Short-term exposure to air pollution can produce various asthma outcomes, such as damaging lung functions, exacerbating asthma symptoms, and increasing emergency-room visits [4].

However, it has been proved that green space structure can reshape air pollution concentrations. On the one hand, the types and proportions of green space components can lead to different abilities of the flora to reduce air pollution; on the other hand, it can influence the spread of air pollution by changing wind direction and speed [5-6]. Given 
that, green space structure has the potential to influence the prevalence of asthma through decreasing air pollution. Besides, green space structure can directly influence asthma via its micro-components. The diverse microbiota within the green space is capable to increase human commensal microbiota on the skin and nasal, which subsequently contributes to enhancing their immunity to allergy atopies [7].

In sum, there are multiple potential mechanisms through which green space structure influence asthma, and the co-effects as mentioned above might jointly work on asthma. Yet, most studies only focus on one effect or using a partial view to explain the mechanism; and some tend to pay more attention to the associations between green space and air pollution, or between air pollution and asthma [4,8-9]. Moreover, although there are a few works has examined the relationship between green space and asthma, the conclusions are inconsistent [10-11]. In this sense, this study takes Toronto as a case to explore two potential mechanisms between green space structure and asthma, namely directly influence on asthma via its micro-component (diverse microbiota), and indirectly impact upon asthma by reducing air pollution.

\section{Materials and Methods}

As discussed above, except for the direct effects of green space structure (independent variable) on the prevalence of asthma (dependent variable), possibly the influence may be mediated by air pollution (mediator variable). That is to say, the study needs to examine the associations between the independent variable and the mediator variable, between the mediator variable and the dependent variable, as well as between the independent variable and the dependent variable. Given that, regression analysis is applied in SPSS 20 v. PROCESS 3.4 (Andrew F. Hayes, Ohio State University, USA) (Downloaded from http://processmacro.org/download.html). It is noteworthy that the indirect pathway hypothesis would not be confirmed if the associations between the independent variable and mediator variable or between the mediator variable and the dependent variable are not statistically significant. All variables are calculated by taking the neighborhood as the statistical unit in this study. Detailed explanation of the variables is as follow.

- Independent variable

The green space structure is measured by the ratio of the tree to shrub and grass (calculation method: tree areas divided by the sum of shrub areas and grass areas), and the data of vegetation types (including trees, shrubs, and grass) is obtained from Toronto Parks, Forestry \& Recreation.

- Dependent variable

The prevalence of asthma is denoted by the number of asthmatics per 100 people and the relevant data is provided by the Ontario Community Health Profiles Partnership. Besides, considering gender difference, this study makes a further analysis to explore whether or not green space structure plays different roles in different gender asthmatics. In other words, the prevalence of male asthmatics and the prevalence of female asthmatics are treated as the dependent variable in the analysis model respectively.

- Mediator variable

Air pollution is described by two indicators which are ultrafine particles and total pollutants released to the air. In particular, the former is obtained from Toronto Social Development, Finance \& Administration, and the latter is derived from the study by Sabaliauskas et al. (2015) [12].

- Moderator variable

Except for the above indicators, the factors that might moderate (enhance/decrease) the influence of green space structure on the prevalence of asthma are also considered in this study. Previous studies have shown that tree species, on the one hand, can make a difference in air pollution [13], on the other hand, could influence asthma by producing pollen and influencing the microbial community [14-15]. That is to say, tree species can possibly exert 
impacts through both direct and indirect pathways. Given that, (street) tree diversity is considered as the moderator and calculated by the Shannon-Wiener index [16]. The data of street tree species is obtained from Toronto Parks, Forestry \& Recreation.

- Covariates

Existing evidence suggests that asthma is a kind of disease caused by multiple aspects [3]. Apart from the factors examined in this study, there are other elements probably influencing the prevalence of asthma. Hence it is necessary to control the influence of these factors on the analysis results. As this study is based on the population but not individuals, therefore, those individual factors are not considered. By referring to existing studies, the percentage of green space, average total income, average household size, and the percentage of the total visible minority are considered as covariates [17-19]. The green space data are provided by Toronto Parks, Forestry \& Recreation and data for the other covariates are all obtained from Toronto Social Development, Finance \& Administration.

\section{Results}

The analysis results are summarized in Table 1 . When taking air pollution as the outcome, the ratio of the tree to shrub and grass (RTSG) is negatively related to ultrafine particles (UFP), while it has no significant relationship with (total) pollutants released to the air (PRA). What's more, tree diversity (TD) can influence neither UFP nor PRA and does not moderate the impacts of RTSG on air pollution in this study. For covariates, contrary to RTSG, the percentage of green space (POGS) contributes to reducing PRA, while its association with UFP is nonsignificant. Intriguingly, total income (TI) positively correlates with UFP, whereas there is no a similar relationship between it and PRA.

When taking the prevalence of asthma as the outcome, RTSG is uncorrelated to any type of the prevalence of asthma (namely, total asthmatics, male asthmatics, and female asthmatics). Similarly, air pollution measured by UFP and PRA was found not to be associated with the prevalence of asthma either. That is to say, the hypothesis that RTSG influences the prevalence of asthma by reducing air pollution is not supported by the evidence presented in this study. TD does not exert a significant influence on developing asthma, however, it can moderate RSTG to make a significantly negative impact on male asthmatics. Notably, this moderation effect does not work on the relationship between RTSG and female asthmatics. For covariates, household size (HS) and the percentage of total visible minority (PTVMP) have statistically significant impacts on the prevalence of asthma, while POGS has no such influence. Moreover, compared with PMA, TI matters more for PFA and it negatively correlates with female asthmatics.

Table 1. Coefficients of variables.

\begin{tabular}{cccccc}
\hline \multirow{2}{*}{ Variables } & \multicolumn{5}{c}{ Outcomes } \\
\cline { 2 - 6 } & \multicolumn{2}{c}{ Air pollution } & \multicolumn{3}{c}{ Prevalence of asthma } \\
& UFP & PRA & PTA & PMA & PFA \\
\hline Constant & 0.018 & -0.002 & -0.032 & -0.032 & -0.032 \\
RTSG & $-0.307^{* *}$ & -0.104 & -0.186 & -0.192 & -0.170 \\
UFP & N/A & N/A & 0.015 & 0.028 & 0.008 \\
PRA & N/A & N/A & 0.013 & 0.017 & 0.006 \\
TD & -0.039 & 0.051 & 0.074 & 0.083 & 0.066 \\
Int_1 & 0.102 & -0.010 & $-0.187^{*}$ & $-0.183^{*}$ & -0.183 \\
POGS & 0.069 & $-0.315^{* *}$ & 0.123 & 0.104 & 0.133 \\
TI & $0.235^{*}$ & 0.161 & $-0.230^{*}$ & -0.108 & $-0.328^{* *}$ \\
HS & 0.202 & 0.216 & $0.464^{* * *}$ & $0.562^{* * *}$ & $0.355^{* * *}$ \\
PTVMP & 0.168 & 0.107 & $-0.527^{* * *}$ & $-0.483^{* * *}$ & $-0.542^{* * *}$ \\
\hline
\end{tabular}


Note: (1) PTA, PMA, and PFA are the abbreviation of the prevalence of total, male and female asthmatics, respectively; RTSG is the abbreviation of the ratio of the tree to shrub and grass; UFP is the abbreviation of ultrafine particles; PRA is the abbreviation of the total pollutants released to the air; TD is the abbreviation of tree diversity; POGS is the abbreviation of the percentage of green space; TI is the abbreviation of (average) total income (in neighborhood-level); HS is the abbreviation of (average) household size (in neighborhood-level); PTVMP is the abbreviation of the percentage of the total visible minority. (2) Int_1 means the interaction between RTSG and TD. (3) ${ }^{*}$ represents $\mathrm{p}<=0.05 ;{ }^{* *}$ represents $\mathrm{p}<=0.01$; ${ }^{* * *}$ represents $\mathrm{p}<=0.001$. (4) Level of confidence for all confidence intervals in output: 95.0000. (5) Dependent variables of PTA, PMA, and PFA are separately taken into the analysis model.

\section{Discussions and Conclusions}

This study explores two potential pathways between RSTG and the prevalence of asthma. In detail, there is no robust evidence to show that the impact of green space structure on the prevalence of asthma is by reducing air pollution, which might be due to the time inconsistence of the data. Although the average change rate of the total pollutants released to the air decreases slightly [20], the time bias among vegetation data (extracted in 2018), asthma data (collected in 2016-2017), UFP data (measured in 2008), and PRA (obtained in 2012) probably causes the difference. Regarding the direct pathway between green space structure and the prevalence of asthma, it is interesting that green space structure has no significant relationship with the prevalence of asthma, but when moderated by tree diversity, it has the potential to reduce the prevalence of male asthmatics and such moderation effect only works on male asthmatics. That might be due to the difference in green space utility between males and females. Previous studies suggest that biodiversity, like trees, shrubs, etc., contributes to enhancing green space use. But compared with the males, females are more easily influenced by safety perception while using the green space [21]. This is probably why males tend to use green space more frequently [22]. As has been revealed, the direct impact of green space structure on the prevalence of asthma is based on the hypothesis that green space micro-component, diverse microbiota, can improve human immunity to allergic atopy by increasing microbial diversity on the skin when they use green space [23]. In other words, only when people physically use green space, then this potential mechanism works. Therefore, when moderated by tree diversity, green space structure can reduce asthma risk for males, but it is not significant for females.

The positive association between household size and asthma as well as the negative relationship between the percentage of total visible minority and asthma echoes with the existing findings $[17,24]$. The nonsignificant correlation between the percentage of green space and asthma in this study resonates with some studies. Yet, it is also found that the negative relationship even the positive relationship between them also exists [25-27], which might be due to the different research geographic context [28]. The different impacts of household income on male and female asthmatics indicate that compared with male asthmatics, female asthmatics are more easily influenced by the economic level and the deprived people tend to have a high risk of developing asthma. But this finding is significant only in female asthmatics.

It is noteworthy that the vegetation types (trees, shrubs, and grass) applied in this study are classified automatically by following certain classification model logic. To reduce the influence of classification bias, this study combines shrubs with grass to calculate the ratio of trees to shrubs and grass, but the difference between the extracted vegetation types and the ones in reality probably still makes a difference in the analysis results. Therefore, it should be well noted while applying the findings as explained above to planning or treatment practice.

Author Contributions: Y.D. and H.L. jointly conceptualized the study and analytical design, conducted the analyses and interpreted the results, writing and reviewing of manuscript drafts; Y.D. and T.Z. collected the data and preprocess them.

Funding: This work was funded by "Ministry of Education, grant number 19GBQY083" and "National Natural Science Foundation of China, grant number D1218006". 
Acknowledgments: Great thanks to the University of Toronto Researchers (Kelly Sabaliauskas and Greg Evans) for providing the data of ultrafine particles applied in this study.

Conflicts of Interest: Declare conflicts of interest or state "The authors declare no conflict of interest."

\section{References}

1 Asthma Canada. Understanding Asthma. https://asthma.ca/get-help/understanding-asthma/

2 Asthma Canada. A snapshot of asthma in Canada: 2019 Annual asthma survey report. 2019.

3World Health Organisation. Asthma. 2020. https://www.who.int/en/news-room/fact-sheets/detail/asthma

4 Tiotiu, A.I.; Novakova, P.; Nedeva, D.; et al. Impact of Air Pollution on Asthma Outcomes. International journal of environmental research and public health 2020,17(17). DOI: 10.3390/ijerph17176212

5 Qiu, L.; Liu, F.; Zhang, X.; Gao, T. Reducing effect of air particulate matter concentration by green spaces with different vegetation structure in city park. Research of Environmental Sciences 2018, 31(10), pp. 1685-1694.

6 Sun, X.D.; Li, H.M.; Liu, X.; Xu, M. Subduction ability of different green space structure on atmospheric particulate matter. Environmental Chemistry 2017, 36(2), pp. 289-295.

7 Rook, G.A. Regulation of the immune system by biodiversity from the natural environment: An ecosystem service essential to health. Proceedings of the national academy of sciences of the united states of America 2013, 110(46), pp. 18360-18367.

8 Rufo, J.C.; Paciencia, I.; Ribeiro, A.I. Green Environments and Allergic Diseases in Children: a Scoping Review. Current epidemiology reports 2019, 6(4), pp. 442-448.

9 Eisenman, T.S.; Churkina, G.; Jariwala, S.P.; et al. Urban trees, air quality, and asthma: An interdisciplinary review. Landscape and urban planning 2019, 187, pp.47-59.

10 Squillacioti, G.; Bellisario, V.; Levra, S.; et al. Greenness Availability and Respiratory Health in a Population of Urbanised Children in North-Western Italy. International journal of environmental research and public health 2020, 17(1), 108. DOI: 10.3390/ijerph17010108

11 Ferrante, G.; Asta, F.; Cilluffo, G.; et al., The effect of residential urban greenness on allergic respiratory diseases in youth: a narrative review. World allergy organization journal 2020,13 (1). DOI: 10.1016/j.waojou.2019.100096

12 Sabaliauskas, K.; Jeong, C.H.; Yao, X.H.; et al. Development of a land-use regression model for ultrafine particles in Toronto, Canada. Atmospheric environment 2015, 110, pp. 84-92.

13 Beckett, K.P.; Freer-Smith, P.; Taylor, G. Effective tree species for local air-quality management. Journal of arboriculture 2000, 26(1), pp. 12-19.

14 Lai, Y.; Kontokosta, C.E. The impact of urban street tree species on air quality and respiratory illness: A spatial analysis of largescale, high-resolution urban data. Health E place 2019, 56, pp. 80-87.

15 Zeng, J.Y.; Vuong, T.M.D.; Zhang, B.W.; et al. An investigation into whether effect of tree species on soil microbial community is related with deciduous property or leaf shape. Catena 2020, 195. DOI: 10.1016/j.catena.2020.104699

16 Shannon, C. E.; Weaver, W. The mathematical theory of communication. Urbana: University of Illinois Press $1963,117$.

17 Trivedi, M.; Denton, E. Asthma in Children and Adults-What Are the Differences and What Can They Tell us About Asthma. Frontiers in pediatrics 2019, 7. DOI: 10.3389/fped.2019.00256

18 Aarab, R.; Vijverberg, S.J.H.; Prins, M.; et al. Prevalence of and factors associated with adult-onset asthma in different ethnic groups: The HELIUS study. Respiratory medicine 2019,150, pp. 113-119.

19 Idani, E.; Raji, H.; Maraghi, E.; et al. Risk factors associated with asthma among adults in Khuzestan, southwest Iran. Clinical epidemiology and global health 2020, 8(2), pp. 350-355.

20 City government, Tracking and Reducing Chemicals in Toronto: Annual ChemTRAC Reports 2012-2018.

21 Foster, C.; Hillsdon, M.; Thorogood, M. Environmental perceptions and walking in English adults. Journal of epidemiology and community health 2004, 58(11), pp. 924-928

22 Schipperijn, J.; Ekholm, O.; Stigsdotter, U.K.; et al. Factors influencing the use of green space: Results from a Danish national representative survey. Landscape and urban planning 2010, 95(3), pp. 130-137.

23 Selway, C.A.; Mills, J.G.; Weinstein, P.; et al. Transfer of environmental microbes to the skin and respiratory tract of humans after urban green space exposure. Environment international 2020, 145. DOI: 10.1016/j.envint.2020.106084

24 Tan D.J.; Walters E.H.; Perret J.L.; et al. Clinical and functional differences between early-onset and late-onset adult asthma: a population-based Tasmanian Longitudinal Health Study. Thorax 2016, 71, pp. 981-987.

25 Dadvand, P.; Villanueva, C.M.; Font-Ribera, L.; et al. Risks and benefits of green spaces for children: a cross-sectional study of associations with sedentary behaviour, obesity, asthma, and allergy. Environmental health perspectives 2014, 122, pp. $1329-1335$.

26 Zeng, X.W.; Lowe, A.J.; Lodge, C.J.; et al. Greenness surrounding schools is associated with lower risk of asthma in schoolchildren. Environment international 2020, 143. DOI: 10.1016/j.envint.2020.105967

27 Andrusaityte, S.; Grazuleviciene, R.; Kudzyte, J.; et al. Associations between neighbourhood greenness and asthma in preschool children in Kaunas, Lithuania: a case-control study. BMJ OPEN 2016, 6(4). DOI: 10.1136/bmjopen-2015-010341

28 Dzhambov, A.M.; Lercher, P.; Rudisser, J.; et al. Allergic symptoms in association with naturalness, greenness, and greyness: A cross-sectional study in schoolchildren in the Alps. Environmental research 2020, In Press. DOI: 10.1016/j.envres.2020.110456. 